\title{
ENDOMORPHISM NEAR-RINGS OF $p$-GROUPS GENERATED BY THE AUTOMORPHISM AND INNER AUTOMORPHISM GROUPS
}

\author{
GARY L. PETERSON
}

(Communicated by Maurice Auslander)

\begin{abstract}
The purpose of this paper is to investigate the equality of the endomorphism near-rings generated by the automorphism group and inner automorphism group of a nonabelian $p$-group $G$. If the automorphism group of $G$ is not a $p$-group, we find that these near-rings are different. If the automorphism group of $G$ is a $p$-group, examples are given illustrating that these near-rings can be different and can be the same.
\end{abstract}

Suppose that $G$ is an additive (but not necessarily abelian) group and $S$ is a semigroup of endomorphisms of $G$. Under pointwise addition and composition of functions, the set $R$ of all functions from $G$ to $G$ of the form $\varepsilon_{1} s_{1}+\cdots+$ $\varepsilon_{n} s_{n}$, where $\varepsilon_{i}= \pm 1$ and $s_{i} \in S$, along with the zero map forms a distributively generated left near-ring when functions are written on the right-hand side. If $S$ is the automorphism group of $G, \operatorname{Aut}(G)$, it is customary to denote this endomorphism near-ring by $A(G)$; if $S$ is the inner automorphism group of $G$, $\operatorname{Inn}(G)$, this endomorphism near-ring is often denoted by $I(G)$. Of course, if $\operatorname{Aut}(G)=\operatorname{Inn}(G)$, the near-rings $A(G)$ and $I(G)$ coincide, but there are many cases where $\operatorname{Aut}(G) \neq \operatorname{Inn}(G)$ and $A(G)=I(G)$. Instances of this occur, for example, because $A(G)=I(G)$ when $G$ is a finite nonabelian simple group (see [1]). Other examples where $A(G)$ and $I(G)$ are the same as well as examples of where they are different can be found in Chapter 11 of [6]. In this paper, we shall investigate the equality of $A(G)$ and $I(G)$ when $G$ is a finite $p$-group ( $p$ a prime). Related to our study, the reader should note that we always have $\operatorname{Aut}(G) \neq \operatorname{Inn}(G)$ when $G$ is a $p$-group of order more than $p$, because $G$ has an outer automorphism of order $p$ by a theorem of Gaschütz [3, III, 19.1].

Suppose now that $G$ is a finite $p$-group. If $G$ is abelian, it is a simple matter to check that $A(G)=I(G)$ if and only if $G$ is cyclic. Consequently, we will restrict our attention to the case when $G$ is nonabelian. Let us first consider the case when $\operatorname{Aut}(G)$ is not a $p$-group. In this case we obtain $A(G) \neq I(G)$. In fact, we can prove an even more general result:

Received by the editors March 30, 1992; presented at the 99th Annual Meeting of the American Mathematical Society, January 14, 1993.

1991 Mathematics Subject Classification. Primary 16A76; Secondary 20D45, 20E36, 20 F28.

Key words and phrases. Endomorphism near-ring, automorphism group, inner automorphism group. 
Theorem. Suppose that $G$ is a nonabelian p-group, $A$ is a group of automorphisms of $G$ containing $\operatorname{Inn}(G)$, and $R$ is the endomorphism near-ring of $G$ generated by $A$. If $A$ is not a p-group, then $R \neq I(G)$.

Proof. Suppose we did have $R=I(G)$. On the one hand, Corollary 3.3 of [4] tells us that $I(G)$ is a local near-ring. Note that we might as well assume that $A=\operatorname{Aut}(G) \cap R$, since $A \subseteq \operatorname{Aut}(G) \cap R$ and $\operatorname{Aut}(G) \cap R$ additively generates $R$. Thus by Theorem 2.3 of [4] we conclude that $A$ has a normal $p$-Sylow subgroup $P$ and that $P$ has a cyclic complement $K$ in $A$. Let $|K|=k$.

On the other hand, let us apply the theorem of [7]. Suppose that $L / H$ is an $R$-principal factor of $G$ (that is, $H \subseteq L$ are $R$-ideals of $G$ such that $L / H$ is a minimal $R$-module) of order $p^{n}$. This theorem tells us that $R$ is not local for an automorphism group of our form when $k$ does not divide $p^{i}+p^{j}-1$ for each $0 \leq i, j \leq n-1$. Of course, if $R=I(G)$, then $L / H$ is a minimal normal subgroup of $G / H$ and so has order $p$. Hence any such $p^{i}+p^{j}-1$ is 1 , which is certainly not divisible by $k$. Thus $R$ is not local, a contradiction.

Now let us consider the case when $\operatorname{Aut}(G)$ is a $p$-group. In this case we can give examples showing that no general conclusion can be drawn. From [5] we see that the dihedral 2-groups furnish us with examples of $p$-groups where Aut $(G)$ is a $p$-group and $A(G) \neq I(G)$. To obtain examples where we do have $A(G)=I(G)$, we shall consider the semidihedral groups. These are groups of order $2^{m}$ with $m>3$ of the form

$$
G=\left\langle a, b \mid 2^{m-1} a=2 b=0,-b+a+b=\left(-1+2^{m-2}\right) a\right\rangle .
$$

We first shall obtain a description of $I(G)$ similar to the one obtained for the dihedral groups in [5]. Some of the work we are about to do may be omitted if we only wish to see that $A(G)=I(G)$ but will be included since this example may be of independent interest.

The inner automorphism of $G$ induced by an element $g \in G$ will be denoted $[g]$, and [0] will be denoted as 1 . Also, we shall indicate a map from $G$ to $G$ by giving the respective images of general elements $n a$ and $n a+b$ of $G$ in a bracketed pair [ , ]. For example,

$$
[k a]=\left[n a,\left(n-2 k+k 2^{m-2}\right) a+b\right]
$$

and

$$
[k a+b]=\left[\left(-n+n 2^{m-2}\right) a,\left(-n+n 2^{m-2}+2 k-k 2^{m-2}\right) a+b\right] .
$$

Let us set

$$
\delta=-1+[a]=\left[0,\left(2-2^{m-2}\right) a\right] \text { and } \alpha=1+[b]=\left[n 2^{m-2} a, 2 n a\right] .
$$

The reader can verify that

$$
[k a]=-k \delta+1 \text { and }[k a+b]=k \delta-1+\alpha,
$$

so that we have $I(G)=\langle 1, \delta, \alpha\rangle$ as additive groups.

Note that $\alpha+\delta=\delta+\alpha$. Also we have $\langle\alpha\rangle \cap\langle\delta\rangle=0$ for suppose $r \alpha=s \delta$, where $r$ and $s$ are integers with $0 \leq r, s<2^{m-2}$. Applying these maps to $2^{m-2} a+b$, we see that $0=s\left(2-2^{m-2}\right) a$. Thus, $s=0$ and hence $\langle\alpha, \delta\rangle=$ $\langle\alpha\rangle \oplus\langle\delta\rangle$. Next, $-1+\alpha+1=-\alpha$ and $-1+\delta+1=-\delta$, so $\langle\alpha, \delta\rangle$ is normal in $I(G)$. Finally, let us verify that $\langle 1\rangle \cap\langle\alpha, \delta\rangle=0$. Suppose that $t=r \alpha+s \delta$, 
where $t, r$, and $s$ are integers with $0 \leq t<2^{m-1}$ and $0 \leq r, s<2^{m-2}$. Applying $t$ and $r \alpha+s \delta$ to $b$, we obtain $t b=s\left(2-2^{m-2}\right) a$, which implies $s=0$ and $2 \mid t$. Applying $t$ and $r \alpha$ to $2 a+b$, which has order 2 , we see that $0=4 r a$, so that $2^{m-3}$ and hence 2 divides $r$. Now applying $t$ and $r \alpha$ to $a$, we have $t a=0$, which forces $t=0$ and hence $r=0$. Thus, $I(G)$ is the semidirect product of $\langle 1\rangle$ and $\langle\alpha, \delta\rangle=\langle\alpha\rangle \oplus\langle\delta\rangle$. Further, $|I(G)|=2^{3 m-5}$.

Let us now turn our attention to $A(G)$. In order to describe the elements of $\operatorname{Aut}(G)$, first note that an element of the form $j a+b$ has order 2 if $j$ is even and order 4 if $j$ is odd. Thus $\langle a\rangle$ is a characteristic subgroup of $G$, and the image of $a$ under an element of $\operatorname{Aut}(G)$ must have the form $i a$, where $\left(i, 2^{m-1}\right)=1$. Also the image of $b$ under an element of $\operatorname{Aut}(G)$ must have the form $j a+b$, where $j$ is even. Conversely, it is a simple matter to check that a mapping sending $a$ to $i a$ and $b$ to $j a+b$ for such $i$ and $j$ extends to an automorphism of $G$. Thus $\operatorname{Aut}(G)$ consists of the maps of the form $[$ in $a,(j+i n) a+b]$, where $\left(i, 2^{m-1}\right)=1$ and $j$ is even. Further, $|\operatorname{Aut}(G)|=\varphi\left(2^{m-1}\right) \cdot 2^{m-2}=2^{2 m-4}$.

Consider the automorphisms

$$
\sigma=[-n a,-n a+b], \quad \beta=[5 n a, 5 n a+b], \quad \tau_{j}=[n a,(j+n) a+b],
$$

where $j$ is even. Since $\sigma$ and $\beta$ restricted to $\langle a\rangle$ generate $\operatorname{Aut}(\langle a\rangle)[2$, Lemma 5.4.1], it follows that the automorphisms $\sigma, \beta$, and $\tau_{j}\left(0<j / 2<2^{m-2}\right)$ generate $\operatorname{Aut}(G)$. Note that $\tau_{j}=\left[(j / 2)\left(-1+2^{m-3}\right) a\right] \in \operatorname{Inn}(G)$. Let us set

$$
\psi=1+\sigma=\left[0,\left(2 n-n 2^{m-2}\right) a\right] .
$$

We then obtain

$$
\alpha=\left(1-2^{m-3}\right)\left(2^{m-2}+\psi\right) .
$$

Solving this equation for $\psi$, we get $\psi$ and hence $\sigma$ in $I(G)$. Further we have

$$
\beta=5-2 \psi
$$

so that $\beta \in I(G)$. Thus $A(G)=I(G)$.

\section{REFERENCES}

1. A. Fröhlich, The near-ring generated by the inner automorphisms of a finite simple group, J. London Math. Soc. 33 (1958), 95-107.

2. D. Gorenstein, Finite groups, Harper and Row, New York, 1968.

3. B. Huppert, Endliche Gruppen. I, Springer-Verlag, Berlin, 1967.

4. C. Lyons and G. Peterson, Local endomorphism near-rings, Proc. Edinburgh Math. Soc. 31 (1988), 409-414.

5. J. Malone and C. Lyons, Finite dihedral groups and d.g. near-rings. II, Compositio Math. 26 (1973), 249-259.

6. J. Meldrum, Near-rings and their links with groups, Pitman, London, 1985.

7. G. Peterson, Automorphism groups emitting local endomorphism near-rings, Proc. Amer. Math. Soc. 105 (1989), 840-843.

Department of Mathematics, James Madison University, Harrisonburg, Virginia

E-mail address: fac-gpeterso@jmuvax1.acs.jmu.edu 